\title{
Generalized Multiplicative Coupled Fibonacci Sequence and its Properties
}

\author{
Omprakash Sikhwal \\ Devanshi Tutorial, Keshaw \\ Kunj, Mandsaur (M.P.), India
}

\author{
Yashwant Vyas \\ Research Scholar, Faculty of \\ Science, Pacific Academy of \\ Higher Education and \\ Research University, Udaipur
}

\author{
Shikha Bhatnagar \\ School of Studies in \\ Mathematics, Vikram \\ University Ujjain, India
}

(Raj.) India

\begin{abstract}
The coupled Fibonacci sequences are new direction of generalization of Fibonacci sequence. The concept of coupled Fibonacci sequence was first introduced by Atanassov, K. T. in 1985. He deliberated multiplicative coupled Fibonacci sequences of second order in 1995. Multiplicative coupled Fibonacci sequences are less known and popularized now days. Generalized Multiplicative Coupled Fibonacci sequences of second order are defined by the recurrence relations $\quad \alpha_{n+2}=p \beta_{n+1} q \beta_{n}$ and $\beta_{n+2}=r \alpha_{n+1} s \alpha_{n}, n \geq 0$ with initial conditions $\alpha_{0}=a, \beta_{0}=b, \alpha_{1}=c, \beta_{1}=d$, where $p, q, r$ and $s$ are real numbers. In this paper, sum identities of generalized multiplicative coupled Fibonacci sequences of second order are presented and derived.
\end{abstract}

\section{Keywords}

Coupled Fibonacci sequences, Multiplicative coupled Fibonacci sequences, Generalized Coupled Fibonacci Sequences.

\section{Mathematics Subject Classification 2010: 11B37, 11B39.}

\section{INTRODUCTION}

The Fibonacci sequence is probably one of the most famous and most widely written about number sequences in all of mathematics. The Fibonacci sequence has been defined by the recurrence relation $F_{n}=F_{n-1}+F_{n-2}, n \geq 2$ with initial conditions $F_{0}=0 \& F_{1}=1$. In the recent years, there has been much interest in development of knowledge in the general region of Fibonacci numbers and related mathematical topics. In last decade, additive coupled Fibonacci sequences are popularized, but multiplicative coupled difference equations or recurrence relations are less known. Now days multiplicative coupled Fibonacci sequences are popularized. This is the new direction of generalization of coupled Fibonacci sequences.

Multiplicative coupled Fibonacci sequences are deliberated in 1995. Firstly Atanassov [1] notified four different schemes in multiplicative form for coupled Fibonacci sequences and new direction of generalization of coupled Fibonacci sequence in [3], [1] and [2]. He was defined and studied about four different ways to generate coupled sequences and called them multiplicative coupled Fibonacci sequences or multiplicative coupled Fibonacci sequences of second order or multiplicative $2-\mathrm{F}$ sequences.

Let $\left\{\alpha_{k}\right\}_{k=0}^{\infty}$ and $\left\{\beta_{k}\right\}_{k=0}^{\infty}$ be two infinite sequences and let $a, b, c$ and $d$ be four arbitrary real numbers with initial

values $\alpha_{0}=a, \beta_{0}=b, \alpha_{1}=c$ and $\beta_{1}=d$. Then four different schemes of multiplicative coupled Fibonacci sequences of second order [2] are as follows:

Scheme First

$\alpha_{n+2}=\beta_{n+1} \beta_{n}, n \geq 0$

$\beta_{n+2}=\alpha_{n+1} \alpha_{n}, n \geq 0$.

Scheme Second

$\alpha_{n+2}=\alpha_{n+1} \beta_{n}, n \geq 0$

$\beta_{n+2}=\beta_{n+1} \alpha_{n}, n \geq 0$.

Scheme Third

$\alpha_{n+2}=\beta_{n+1} \alpha_{n}, n \geq 0$

$\beta_{n+2}=\alpha_{n+1} \beta_{n}, n \geq 0$.

Scheme Fourth

$\alpha_{n+2}=\alpha_{n+1} \alpha_{n}, n \geq 0$

$\beta_{n+2}=\beta_{n+1} \beta_{n}, n \geq 0$.

Singh, B. et al. [10], present coupled Fibonacci sequence of fifth order with some properties for positive and negative integers. Singh, B. and Sikhwal, O. [11], present fundamental properties of Multiplicative coupled Fibonacci sequences of second order. Rathore, G. P. S. et al. [8] presents Multiplicative coupled Fibonacci sequences of third order under two specific schemes. Rathore G. P. S. [9] et al. presents generalized coupled Fibonacci sequences of second order.

\section{GENERALIZED MULTIPLICATIVE COUPLED FIBONACCI SEQUENCES}

Atanassov, K. T. was introduced new generalized coupled Fibonacci sequence. Let $\left\{\alpha_{k}\right\}_{k=0}^{\infty}$ and $\left\{\beta_{k}\right\}_{k=0}^{\infty}$ be two infinite sequences and let $a, b, c$ and $d$ be four arbitrary real numbers with initial values $\alpha_{0}=a, \beta_{0}=b, \alpha_{1}=c$ and $\beta_{1}=d$. Then four different schemes of second order generalized Multiplicative coupled Fibonacci sequences [4] are defined as follows:

Scheme First
$\begin{aligned} & \alpha_{n+2}=p \beta_{n+1} q \beta_{n}, n \geq 0 \\ & \beta_{n+2}=r \alpha_{n+1} s \alpha_{n}, n \geq 0 .\end{aligned}$ 
$\alpha_{n+2}=r \alpha_{n+1} q \beta_{n}, n \geq 0$

$\beta_{n+2}=p \beta_{n+1} s \alpha_{n}, n \geq 0$.

\section{Scheme Third}

$\alpha_{n+2}=p \beta_{n+1} s \alpha_{n}, n \geq 0$

$\beta_{n+2}=r \alpha_{n+1} q \beta_{n}, n \geq 0$.

\section{Scheme Fourth}

$\alpha_{n+2}=r \alpha_{n+1} s \alpha_{n}, n \geq 0$

$\beta_{n+2}=p \beta_{n+1} q \beta_{n}, n \geq 0$.

where $p, q, r$ and $s$ are real numbers.

Now we present some properties of generalized multiplicative coupled Fibonacci sequences of second order under first and Second schemes.

\section{PROPERTIES OF SCHEME FIRST}

First few terms of scheme first (2.1) are as follows:

\begin{tabular}{|l|l|l|}
\hline$n$ & $\alpha_{n}$ & $\beta_{n}$ \\
\hline 0 & $a$ & $b$ \\
\hline 1 & $c$ & $d$ \\
\hline 2 & $p q b d$ & $r s a c$ \\
\hline 3 & $p q r s a c d$ & $p q r s b c d$ \\
\hline 4 & $p^{2} q^{2} r^{2} s^{2} a b c^{2} d$ & $p^{2} q^{2} r^{2} s^{2} a b c d^{2}$ \\
\hline 5 & $p^{4} q^{4} r^{3} s^{3} a b^{2} c^{2} d^{3}$ & $p^{3} q^{3} r^{4} s^{4} a^{2} b c^{3} d^{2}$ \\
\hline 6 & $p^{6} q^{6} r^{6} s^{6} a^{3} b^{2} c^{4} d^{4}$ & $p^{6} q^{6} r^{6} s^{6} a^{2} b^{3} c^{4} d^{4}$ \\
\hline 7 & $p^{10} q^{10} r^{10} s^{10} a^{4} b^{4} c^{7} d^{6}$ & $p^{10} q^{10} r^{10} s^{10} a^{4} b^{4} c^{6} d^{7}$ \\
\hline
\end{tabular}

Theorem (3.1). For every integer $n \geq 0$,
(a) $\beta_{0} \alpha_{3 n+3}=\alpha_{0} \beta_{3 n+3}$,
(b) $\beta_{1} \alpha_{3 n+4}=\alpha_{1} \beta_{3 n+4}$,
(c) $\beta_{2} \alpha_{3 n+5}=\alpha_{2} \beta_{3 n+5}$.

Proof. (a) To prove this, we shall use mathematical induction method.

If $n=0$, then

$\beta_{0} \alpha_{3}=\beta_{0}\left(p \beta_{2} q \beta_{1}\right)$,

(By scheme 2.1)

$=\beta_{0} p\left(r \alpha_{1} s \alpha_{0}\right) q \beta_{1}$,

(By scheme 2.1)

$=p \beta_{1} q \beta_{0}\left(r \alpha_{1} s \alpha_{0}\right)$,

$=\alpha_{2}\left(r \alpha_{1} s \alpha_{0}\right)$,

(By scheme 2.1)

$=\left(r \alpha_{2} s \alpha_{1}\right) \alpha_{0}$,

$=\beta_{3} \alpha_{0}$.

(By scheme 2.1)

Thus the result is true for $n=0$.

Let us assume that the result is true for some integer $n \geq 1$, then

$\beta_{0} \alpha_{3 n+6}=\beta_{0} \alpha_{3 n+6}$,

(By scheme 2.1)

$=\beta_{0}\left(p \beta_{3 n+5} q \beta_{3 n+4}\right)$,

(By scheme 2.1) $=\beta_{0} p\left(r \alpha_{3 n+4} s \alpha_{3 n+3}\right) q \beta_{3 n+4}$,

(By scheme 2.1)

$=p\left(r \alpha_{3 n+4} s\left(\beta_{0} \alpha_{3 n+3}\right)\right) q \beta_{3 n+4}$,

$=p\left(r \alpha_{3 n+4} s\left(\alpha_{0} \beta_{3 n+3}\right)\right) q \beta_{3 n+4}$,

(By hypothesis)

$=\alpha_{0} \alpha_{3 n+4}\left(p \beta_{3 n+4} q \beta_{3 n+3}\right) r s$,

$=\alpha_{0} \alpha_{3 n+4}\left(\alpha_{3 n+5}\right) r s$,

(By scheme 2.1)

$=\alpha_{0} \beta_{3 n+6}$.

(By scheme 2.1)

Hence by mathematical induction method results is true for all integers $n \geq 0$.

Similarly $(b)$ and (c) can be derived by mathematical induction method.

Theorem (3.2). For every integer $n \geq 0$

$\alpha_{n+4}=p q r^{2} s^{2} \alpha_{n+2} \alpha_{n+1}^{2} \alpha_{n}$ and $\beta_{n+4}=p^{2} q^{2} r s \beta_{n+2} \beta_{n+1}^{2} \beta_{n}$.

Proof. To prove this, we shall use mathematical induction method.

If $n=0$, then

$\alpha_{4}=p q r^{2} s^{2} \alpha_{2} \alpha_{1}^{2} \alpha_{0}$,

$=p q r^{2} s^{2}\left(\alpha_{2} \alpha_{1} \alpha_{1} \alpha_{0}\right)$,

$=p q r^{2} s^{2}\left(\frac{\beta_{3}}{r s} \frac{\beta_{2}}{r s}\right)$,

(By scheme 2.1)

$=p q r^{2} s^{2}\left(\frac{\alpha_{4}}{p q r^{2} s^{2}}\right)$,

(By scheme 2.1)

$=\alpha_{4}$.

Also, $\beta_{4}=p^{2} q^{2} r s \beta_{2} \beta_{1}^{2} \beta_{0}$,

$=p^{2} q^{2} r s\left(\beta_{2} \beta_{1} \beta_{1} \beta_{0}\right)$,

$=p^{2} q^{2} r s\left(\frac{\alpha_{3}}{p q} \frac{\alpha_{2}}{p q}\right)$,

(By scheme 2.1)

$=p^{2} q^{2} r s\left(\frac{\beta_{4}}{p^{2} q^{2} r s}\right)$

(By scheme 2.1)

$=\beta_{4}$.

Thus the result is true for $n=0$.

Now assume that result is true for some integer $n \geq 1$, then

$\alpha_{n+5}=p \beta_{n+4} q \beta_{n+3}$,

(By scheme 2.1)

$=p\left(p^{2} q^{2} r s \beta_{n+2} \beta_{n+1}^{2} \beta_{n}\right) q\left(p^{2} q^{2} r s \beta_{n+1} \beta_{n}^{2} \beta_{n-1}\right)$, (By scheme 2.1)

$=p q r^{2} s^{2}\left(p \beta_{n+2} q \beta_{n+1} p \beta_{n+1} q \beta_{n}\right)\left(p \beta_{n+1} q \beta_{n} p \beta_{n} q \beta_{n-1}\right)$,

$=p q r^{2} s^{2} \alpha_{n+3} \alpha_{n+2} \alpha_{n+2} \alpha_{n+1}$,

(By scheme 2.1)

$=p q r^{2} s^{2} \alpha_{n+3} \alpha_{n+2}^{2} \alpha_{n+1}$.

(By scheme 2.1)

Also, $\beta_{n+5}=r \alpha_{n+4} s \alpha_{n+3}$,

(By scheme 2.1)

$=r\left(p q r^{2} s^{2} \alpha_{n+2} \alpha_{n+1}^{2} \alpha_{n}\right) s\left(p q r^{2} s^{2} \alpha_{n+1} \alpha_{n}^{2} \alpha_{n-1}\right)$, (By scheme 2.1)

$=p^{2} q^{2} r s\left(r \alpha_{n+2} s \alpha_{n+1} r \alpha_{n+1} s \alpha_{n}\right)\left(r \alpha_{n+1} s \alpha_{n} r \alpha_{n} s \alpha_{n-1}\right)$,

$=p^{2} q^{2} r s \beta_{n+3} \beta_{n+2} \beta_{n+2} \beta_{n+1}$,

(By scheme 2.1)

$=p^{2} q^{2} r s \beta_{n+3} \beta_{n+2}^{2} \beta_{n+1}$.

(By scheme 2.1)

Hence by mathematical induction method result is true for all integers $n \geq 0$

\section{PROPERTIES OF SCHEME SECOND}

First few terms of scheme second (2.2) are as follows: 


\begin{tabular}{|l|l|l|}
\hline$n$ & $\alpha_{n}$ & $\beta_{n}$ \\
\hline 0 & $a$ & $b$ \\
\hline 1 & $c$ & $d$ \\
\hline 2 & $r q b c$ & $p s a d$ \\
\hline 3 & $r^{2} q^{2} b c d$ & $p^{2} s^{2} a c d$ \\
\hline 4 & $r^{3} q^{3} p s a b c d^{2}$ & $p^{3} s^{3} r q a b c^{2} d$ \\
\hline 5 & $r^{4} q^{4} p^{3} s^{3} a^{2} b c^{2} d^{3}$ & $p^{4} s^{4} r^{3} q^{3} a b^{2} c^{3} d^{2}$ \\
\hline 6 & $p^{6} q^{6} r^{6} s^{6} a^{3} b^{2} c^{4} d^{4}$ & $p^{6} q^{6} r^{6} s^{6} a^{2} b^{3} c^{4} d^{4}$ \\
\hline 7 & $p^{10} q^{10} r^{10} s^{10} a^{4} b^{4} c^{7} d^{6}$ & $p^{10} q^{10} r^{10} s^{10} a^{4} b^{4} c^{6} d^{7}$ \\
\hline 8 & $r^{17} q^{17} p^{16} s^{16} a^{6} b^{7} c^{11} d^{10}$ & $p^{17} s^{17} r^{16} q^{16} a^{7} b^{6} c^{10} d^{11}$ \\
\hline 9 & $r^{28} q^{28} p^{26} s^{26} a^{10} b^{11} c^{17} d^{17}$ & $p^{28} s^{28} r^{26} q^{26} a^{11} b^{10} c^{17} d^{17}$ \\
& & \\
\hline & & \\
\hline
\end{tabular}

Theorem (4.1). For every integer $n \geq 0$ :
(a) $\beta_{0} \alpha_{6 n}=\alpha_{0} \beta_{6 n}$,
(b) $\beta_{1} \alpha_{6 n+1}=\alpha_{1} \beta_{6 n+1}$,
(c) $\beta_{2} \alpha_{6 n+2}=\alpha_{2} \beta_{6 n+2}$.

Proof. (a) To prove this, we shall use mathematical induction method.

If $n=0$, then

$\beta_{0} \alpha_{0}=\alpha_{0} \beta_{0}$

Thus, result is true for $n=0$.

Now assume that result is true for some integer $n \geq 1$, then

$\beta_{0} \alpha_{6 n+6}=\beta_{0}\left(r \alpha_{6 n+5} q \beta_{6 n+4}\right)$,

(By scheme 2.2)

$=\beta_{0} r\left(r \alpha_{6 n+4} q \beta_{6 n+3}\right) q \beta_{6 n+4}$,

(By scheme 2.2)

$=r^{2} q^{2} \beta_{0} \alpha_{6 n+4} \beta_{6 n+3} \beta_{6 n+4}$,

$=r^{2} q^{2} \beta_{0} \alpha_{6 n+4}\left(p \beta_{6 n+2} s \alpha_{6 n+1}\right) \beta_{6 n+4}$,

(By scheme 2.2)

$=r^{2} q^{2} p s \beta_{0} \alpha_{6 n+4}\left(p \beta_{6 n+1} s \alpha_{6 n}\right) \alpha_{6 n+1} \beta_{6 n+4}$, (By scheme 2.2)

$=r^{2} q^{2} p^{2} s^{2} \alpha_{6 n+4} \beta_{6 n+1}\left(\beta_{0} \alpha_{6 n}\right) \alpha_{6 n+1} \beta_{6 n+4}$,

$=r^{2} q^{2} p^{2} s^{2} \alpha_{6 n+4} \beta_{6 n+1}\left(\alpha_{0} \beta_{6 n}\right) \alpha_{6 n+1} \beta_{6 n+4}$, (By hypothesis)

$=r q p^{2} s^{2} \alpha_{0} \alpha_{6 n+4} \beta_{6 n+1} \alpha_{6 n+2} \beta_{6 n+4}$,

(By scheme 2.2)

$=p^{2} s^{2} \alpha_{0} \alpha_{6 n+4} \alpha_{6 n+3} \beta_{6 n+4}$,

(By scheme 2.2)

$=p s \alpha_{0} \beta_{6 n+5} \alpha_{6 n+4}$,

(By scheme 2.2)

$=\alpha_{0} \beta_{6 n+6}$.

(By scheme 2.2)

Hence by mathematical induction method result is true for all integers $n \geq 0$.

Similarly (b) and (c) can be derived by mathematical induction method.

Theorem (4.2). for every integer $n \geq 0$ : (a) $p^{2} s^{2} \alpha_{0} \alpha_{6 n+3}=r^{2} q^{2} \beta_{0} \beta_{6 n+3}$,

(b) $p^{2} s^{2} \alpha_{1} \alpha_{6 n+4}=r^{2} q^{2} \beta_{1} \beta_{6 n+4}$,

(c) $p^{2} s^{2} \alpha_{2} \alpha_{6 n+5}=r^{2} q^{2} \beta_{2} \beta_{6 n+5}$.

Proof. (a) To prove this, we shall use induction method. If $n=0$ then

$$
\begin{aligned}
& p^{2} s^{2} \alpha_{0} \alpha_{3}=p^{2} s^{2} \alpha_{0}\left(r \alpha_{2} q \beta_{1}\right) \text {, } \\
& \text { (By scheme 2.2) } \\
& =p^{2} s^{2} \alpha_{0} r\left(r \alpha_{1} q \beta_{0}\right) q \beta_{1} \text {, } \\
& \text { (By scheme 2.2) } \\
& =p s r^{2} q^{2} \beta_{2} \alpha_{1} \beta_{0} \text {, } \\
& \text { (By scheme 2.2) } \\
& =r^{2} q^{2} \beta_{3} \beta_{0} \text {. } \\
& \text { (By scheme 2.2) }
\end{aligned}
$$

Thus, result is true for $n=0$

Now assume that result is true for some integer $n \geq 1$, then $p^{2} s^{2} \alpha_{0} \alpha_{6 n+9}=p^{2} s^{2} \alpha_{0}\left(r \alpha_{6 n+8} q \beta_{6 n+7}\right), \quad$ (By scheme 2.2)

$=p^{2} s^{2} r q \alpha_{0}\left(r \alpha_{6 n+7} q \beta_{6 n+6}\right) \beta_{6 n+7}$,

(By scheme 2.2)

$=p^{2} s^{2} r^{2} q^{2} \alpha_{0} \alpha_{6 n+7}\left(p \beta_{6 n+5} s \alpha_{6 n+4}\right) \beta_{6 n+7}$,

(By scheme 2.2)

$=p^{3} s^{3} r^{2} q^{2} \alpha_{0} \alpha_{6 n+7}\left(p \beta_{6 n+4} s \alpha_{6 n+3}\right) \alpha_{6 n+4} \beta_{6 n+7}, \quad$ (By scheme 2.2)

$=p^{2} s^{2} r^{2} q^{2} \alpha_{6 n+7} \beta_{6 n+4}\left(p^{2} s^{2} \alpha_{0} \alpha_{6 n+3}\right) \alpha_{6 n+4} \beta_{6 n+7}$,

$=p^{2} s^{2} r^{2} q^{2} \alpha_{6 n+7} \beta_{6 n+4}\left(r^{2} q^{2} \beta_{0} \beta_{6 n+3}\right) \alpha_{6 n+4} \beta_{6 n+7}$, (By hypothesis)

$=p^{2} s^{2} r^{3} q^{3} \beta_{0} \alpha_{6 n+7} \beta_{6 n+4} \alpha_{6 n+5} \beta_{6 n+7}$,

(By scheme 2.2)

$=p^{2} s^{2} r^{2} q^{2} \beta_{0} \alpha_{6 n+7} \alpha_{6 n+6} \beta_{6 n+7}$,

(By scheme 2.2)

$=p s r^{2} q^{2} \beta_{0} \alpha_{6 n+7} \beta_{6 n+8}$,

(By scheme 2.2)

$=r^{2} q^{2} \beta_{0} \beta_{6 n+9}$.

(By scheme 2.2)

Hence, by mathematical induction method result is true for all integers $n \geq 0$.

Similarly (b) and (c) can be derived by mathematical induction method.

Theorem (4.3). For every integer $n \geq 0$ :

(a) $\alpha_{n+4}=r^{2} q^{2} p s \alpha_{n+2} \beta_{n+1}^{2} \alpha_{n}$,

(b) $\beta_{n+4}=p^{2} s^{2} r q \beta_{n+3} \alpha_{n+2}^{2} \beta_{n+1}$.

Proof. (a) To prove this, we shall use mathematical induction method.

If $n=0$ then

$\alpha_{4}=r^{2} q^{2} \operatorname{ps}_{2} \beta_{1}^{2} \alpha_{0}$,

$=r^{2} q^{2} p s\left(\alpha_{2} \beta_{1} \beta_{1} \alpha_{0}\right)$,

$=r^{2} q^{2} p s\left(\frac{\alpha_{3}}{r q} \frac{\beta_{2}}{p s}\right)$,

(By scheme 2.2)

$=r^{2} q^{2} p s\left(\frac{\alpha_{4}}{r^{2} q^{2} p s}\right)$

(By scheme 2.2)

$=\alpha_{4}$

Thus the result is true for $n=0$.

Now assume that result is true for some integer $n \geq 1$, then

$\alpha_{n+5}=r \alpha_{n+4} q \beta_{n+3}$,

(By scheme 2.2)

$=r\left(r^{2} q^{2} p s \alpha_{n+2} \beta_{n+1}^{2} \alpha_{n}\right) q \beta_{n+3}$,

(By hypothesis)

$=r^{2} q^{2} p s\left(r \alpha_{n+2} q \beta_{n+1}\right)\left(\beta_{n+1} \alpha_{n}\right)\left(p \beta_{n+2} s \alpha_{n+1}\right), \quad$ (By scheme 2.2)

$=r^{2} q^{2} p s\left(\alpha_{n+3}\right)\left(\beta_{n+2}\right)\left(\beta_{n+2} \alpha_{n+1}\right)$,

(By scheme 2.2) 
$=r^{2} q^{2} p s \alpha_{n+3} \beta_{n+2}^{2} \alpha_{n+1}$.

(By scheme 2.2)

Hence, by mathematical induction method result is true for all integers $n \geq 0$.

Proof. (b) To prove this, we shall use mathematical induction method.

If $n=0$ then

$\beta_{4}=p^{2} s^{2} r q\left(\beta_{2} \alpha_{1}^{2} \beta_{0}\right)$,

$=p^{2} s^{2} r q\left(\beta_{2} \alpha_{1} \alpha_{1} \beta_{0}\right)$,

$=p^{2} s^{2} r q\left(\frac{\beta_{3}}{p s} \frac{\alpha_{2}}{r q}\right)$,

(By scheme 2.2)

$=p^{2} s^{2} r q\left(\frac{\beta_{4}}{p^{2} s^{2} r q}\right)$,

(By scheme 2.2)

$=\beta_{4}$.

Thus the result is true for $n=0$.

Now assume that result is true for some integer $n \geq 1$, then

$\beta_{n+5}=p \beta_{n+4} s \alpha_{n+3}$,

(By scheme 2.2)

$=p\left(p^{2} s^{2} r q \beta_{n+2} \alpha_{n+1}^{2} \beta_{n}\right) s \alpha_{n+3}$,

(By hypothesis)

$=p^{2} s^{2} r q\left(p \beta_{n+2} s \alpha_{n+1}\right)\left(\alpha_{n+1} \beta_{n}\right)\left(r \alpha_{n+2} q \beta_{n+1}\right), \quad$ (By scheme 2.2)

$=p^{2} s^{2} r q\left(\beta_{n+3}\right)\left(\alpha_{n+2}\right)\left(\alpha_{n+2} \beta_{n+1}\right)$,

(By scheme 2.2)

$=p^{2} s^{2} r q \beta_{n+3} \alpha_{n+2}^{2} \beta_{n+1}$,

(By scheme 2.2)

Hence, by mathematical induction method result is true for all integers $n \geq 0$.

\section{CONCLUSION}

In this paper, generalized multiplicative coupled Fibonacci sequences of second order are introduced and some properties under specific schemes are discussed and proved these by mathematical induction method.

\section{ACKNOWLEDGMENTS}

The authors are thankful to the reviewers for their constructive suggestions and comments for improving the exposition of the original version.

\section{REFERENCES}

[1] Atanassov, K T., Atanassov V., Shannon A. and Turner J., 2002. New Visual Perspective On Fibonacci Number, World Scientific Publishing Company, Singapore.

[2] Atanassov, K.T., Hlebarova, J. and Mihov, S., 1992. Recurrent formulas of the generalized Fibonacci and Tribonacci sequence, The Fibonacci Quarterly, Vol.30, No. 1, 77-79.

[3] Atanassov, K.T., 1986. On a generalization of the Fibonacci sequence in the case of three sequences, The Fibonacci Quarterly, Vol. 27, No. 1, 7-10.

[4] Atanassov, K. T., 1995. Remark on a New Direction for a Generalization of the Fibonacci Sequence, The Fibonacci Quarterly, Vol. 33, No. 3, 249-250.

[5] Hope, P., 1995. Exponential Growth of Random Fibonacci Sequences, The Fibonacci Quarterly, Vol. 33, No. 2, 164-168.

[6] Glaister, P., 1994. Multiplicative Fibonacci Sequences, The Mathematical Gazette, Vol. 78, No. 481, 68.

[7] Lee, J. Z., Lee, J. S., 1987. Some Properties of the Generalization of the Fibonacci Sequence, The Fibonacci Quarterly, Vol. 25, No.2, 111-117.

[8] Rathore G. P. S., Jain. S. and Sikhwal, O., 2012. Multiplicative Coupled Fibonacci Sequences of third Order, International Journal of Contemporary Mathematical Sciences, Vol. 7, No. 31, 1535 - 1540.

[9] Rathore, G. P. S., Sikhwal, O. and Jain, S., 2012. Generalized Coupled Fibonacci Sequences, International Journal of Computer Application , Vol. 59, No.8, 12-15.

[10] Singh, B., Sikhwal, O., Jain S., 2010. Coupled Fibonacci Sequence of Fifth Order and Some Properties, International Journal of Mathematical Analysis, Vol. 4, No. 25, 1247-1254.

[11] Singh, B. and Sikhwal, O., 2010. Multiplicative Coupled Fibonacci Sequences and Some Fundamental Properties, International Journal of Contemporary Mathematical Sciences, Vol. 5, No. 5, 223-230. 